\title{
One-step processing of plasticized starch/cellulose nanofibrils nanocomposites via twin-screw extrusion of starch and cellulose fibers
}

\author{
Yesmine Fourati $^{\mathrm{a}}$, Albert Magnin ${ }^{\mathrm{b}}$, Jean-Luc Putaux ${ }^{\mathrm{c}}$, Sami Boufi ${ }^{\mathrm{a},{ }^{*}}$ \\ ${ }^{a}$ University of Sfax - LMSE - Faculty of Science - BP 802 - 3018 Sfax, Tunisia \\ ${ }^{b}$ Univ. Grenoble Alpes, CNRS, Grenoble INP, LRP, F-38000 Grenoble, France \\ ${ }^{c}$ Univ. Grenoble Alpes, CNRS, CERMAV, F-38000 Grenoble, France \\ * Corresponding author: sami.boufi@fss.rnu.tn
}

Published in: Carbohydrate Polymers 229 (2020), 115554

DOI: 10.1016/j.carbpol.2019.115554 


\begin{abstract}
Nanocomposites based on thermoplasticized starch filled with cellulose nanofibrils (CNFs) were produced in a single step by twin-screw extrusion of corn starch granules, glycerol as a plasticizer, and oxidized cellulose fibers. The objective was to demonstrate the possibility to produce CNFs in situ during the processing of the nanocomposite when a hydrophilic polymer matrix was used. For comparison purpose, nanocomposites were also prepared by extrusion of a previously prepared CNF suspension, corn starch granules and glycerol. The nanocomposites were characterized in terms of mechanical properties, morphology, crystallinity, and transparency. The nanocomposites prepared via in situ fibrillation displayed a higher strength than those produced by incorporating readily prepared CNFs. In addition, the transparency degree up to a $15 \mathrm{wt} \% \mathrm{CNF}$ content was similar for the two processing routes, confirming the effective breakdown of pretreated cellulose fibers into CNFs during the extrusion process.
\end{abstract}

Keywords: Nanocomposites, cellulose nanofibrils, thermoplastic starch, extrusion 


\section{Introduction}

During these last two decades, extensive work and progress have been accomplished on the production of cellulose nanofibrils (CNFs) and their potential applications in nanostructured biobased materials (Nechyporchuk, Belgacem \& Bras, 2016). The different methods of production without using organic solvents, exceptional intrinsic mechanical properties (Lee et al., 2014), biodegradability (Vikman et al., 2015), lack of safety concerns (Alexandrescu et al., 2013) and high aspect ratio are among the attributes driving for this growing interest.

CNFs have the potential to improve the performance of many products and to replace nonrenewable materials with cellulose. CNFs can be used as reinforcing additive in a wide range of matrices, such as polymers, papers and hydrogels (Boufi et al., 2016).In polymers, a strong reinforcing effect is brought by the addition of CNFs, the magnitude of which depends on (i) the processing route, (ii) type of polymer matrix, (iii) elasticity of the matrix, (iv) degree of dispersion of CNFs and (v)the functionality of their surface (Dufresne, 2017). In paper, CNFs can be added either in bulk or on the surface to improve strength or impart barrier properties (Tarrés et al., 2016). CNFs can also act as a precursor to develop totally new products in value-added markets such as aerogels for biomedical application (Barhoum et al., 2018).

So far, the most popular route for the production of CNFs is based on the high shear mechanical disintegration of the cellulose fibers using high pressure homogenization (HPH) (Besbes, Alila \& Boufi, 2011), microfluidization and wet grinding (Nechyporchuk, Pignon \& Belgacem, 2015). Other methods have been reported, including ultrasonication, high-speed disintegration (Boufi \& Chaker, 2016) and twin-screw extrusion (TSE) (Ho, Abe, Zimmermann \& Yano, 2015; Rol et al., 2017; Baati et al., 2018). Each of these methods has its own advantages and drawbacks and results in the formation of CNF gels with different physical properties in terms of gel viscosity, CNF morphology, transparency, nanosized fraction and consistency. The energy consumption also differs from one process to another with the highest being HPH while the least energy consuming is TSE (Rol et al., 2017).

Recently, TSE revealed to be an effective method for the continuous production of CNFs at high consistency with a solid content ranging from 10 to $30 \mathrm{wt} \%$ (Ho, Abe, Zimmermann \& Yano, 2015; Rol et al., 2017; Baati, Magnin, \& Boufi, 2017, 2018). However, a chemical pretreatment is a prerequisite to facilitate the breakdown of the cellulose fibers. TSE opens the way toward a cost-effective approach for the production of CNFs with a high solid content, low-energy demand and in a continuous way. By contrast to HPH, where the shear rate generated during the injection of the cellulose suspension through the orifice is in the 
order of $10^{5}-10^{6} \mathrm{~s}^{-1}$, the shear rate in the compounding zone in TSE is of the order of 200-500 $\mathrm{s}^{-1}$ for a screw speed up to $400 \mathrm{rpm}$.

The mixing action in twin-screw extruders is based on an elongational flow, where intensive shear and elongation stresses are transferred to fibers as the material is forced to drag through the small gap between screws (typically of the order of a few microns). Accordingly, the viscosity of the continuous phase affects the fibrillation efficiency of the CNF suspension. Replacing water with a polymer solution or suspension such as starch to increase the viscosity of the continuous phase is thus worth investigating.

Thermoplastic starch (TPS) is a class of thermoplastic polymers produced by meltplasticization of starch with a plasticizer such as glycerol, sorbitol, urea or water (Averous, 2016). This class of biobased materials has attracted much attention due to its thermoplasticlike processability, low price and biodegradability. However, TPS is water sensitive, causing properties to change according to the relative humidity of the environment, and exhibits a low mechanical strength compared to commercial commodity plastics. Inclusion of CNFs into TPS thus appears to be an alternative to boost the mechanical properties of the material without impeding its biodegradability. Numerous works have focused on the production and properties of TPS/CNF nanocomposites (Dufresne \& Vignon, 1998; Hietala, Mathew \& Oksman, 2013). Most of them have pointed out a positive impact of the inclusion of CNFs on the mechanical properties in terms of strength and modulus. For instance, nanocomposites were prepared by mixing CNFs extracted from wheat straw using steam explosion, acidic treatment and high-shear mechanical treatment and glycerol and gelatinized starch (Kaushik, Singh \& Verma, 2010). Dynamic mechanical analysis (DMA) and tensile testing showed marked improvement of the tensile modulus and strength at break, confirming the reinforcing effect brought by CNFs. The inclusion of $5 \mathrm{wt} \%$ bacterial cellulose in TPS by melt-mixing brings about a strong reinforcing effect (Martins et al., 2009), with 30- and 6-fold increases in tensile modulus and strength, respectively. Nanocomposite films incorporating CNFs in TPS plasticized with sorbitol were produced by twin-screw extrusion with a CNF content up to 20 wt\% (Hietala, Mathew \& Oksman, 2013). Both tensile strength and modulus were improved, while the strain at break markedly decreased. However, Teixeira et al. (2009) reported that no reinforcing effect was noted with the inclusion of CNFs from cassava bagasse up to $20 \mathrm{wt} \%$ CNF content and the elongation at break was further increased in the presence of glycerol as a plasticizer. The hypothesis of transcrystallization of amylopectin on nanofibrils surface was put forward to account for the loss of the reinforcing effect of the nanocellulose. 
The aim of the present work was to produce nanocomposites based on TPS and CNFs in one step using starch granules, oxidized cellulose fibers, glycerol, and water. Two approaches were used to process the nanocomposites: the first one was the classic melt-mixing of already prepared CNFs with starch granules, glycerol and water, and the second one was the one-pot route where starch granules, glycerol, water and cellulose fibers were extruded together. The breakdown of the cellulose fibers into CNFs took place in situ during the extrusion process and the plasticization of starch with glycerol and water. The mechanical properties, morphology, crystallinity and transparency of the resulting materials prepared by each route were characterized and compared in order to evaluate the relevance of producing nanocomposites based on TPS and CNFs by TSE in one step.

\section{Materials and methods}

\subsection{Materials}

Never-dried Kraft softwood eucalyptus pulp with $50 \mathrm{wt} \%$ water content, kindly provided by Torraspapel S.A. (Spain), was used as a starting material to prepare cellulose nanofibers. Corn starch was kindly provided by Roquette S.A. (Lestrem, France). Sodium chlorite $\left(\mathrm{NaClO}_{2}\right)$, acetic acid (AA) 2,2,6,6-tetramethylpiperidine-1-oxyl radical (TEMPO) and sodium bromide $(\mathrm{NaBr})$ were from Aldrich and used as received. A sodium hypochlorite solution $(\mathrm{NaClO})$ with $4 \%$ chlorine content was a commercial product.

\subsection{TEMPO-mediated oxidation}

The TEMPO-mediated oxidation has been carried out at $\mathrm{pH} 10$ following the method reported in our previous work (Besbes, Alila \& Boufi, 2011). In brief, $400 \mathrm{mg}$ of TEMPO and $3 \mathrm{~g}$ of $\mathrm{NaBr}$ were added to $20 \mathrm{~g}$ of fibers dispersed in $2000 \mathrm{~mL}$ of water. Then, $500 \mathrm{~mL}$ of a $\mathrm{NaClO}$ solution ( $4 \mathrm{wt} \%$ ) was added dropwise to the suspension kept at a temperature around $5{ }^{\circ} \mathrm{C}$. $\mathrm{NaOH}$ solution $(0.01 \mathrm{M})$ was added intermittently to maintain the $\mathrm{pH}$ at around 10 . The oxidized fibers were recovered by filtration using $10 \mu \mathrm{m}$ polyamide bolting cloth and washing with water until the conductivity of the suspension became lower than $500 \mu \mathrm{s}_{\mathrm{m}} \mathrm{m}^{-1}$. The carboxyl content of the fibers was found to be around $740 \mu$ mol.g ${ }^{-1}$. The formation of carboxyl groups was evidenced by the FTIR spectrum that showed a large band at $1600 \mathrm{~cm}^{-1}$ typical of carboxylate, while this band was not observed for the neat fibers (Supplementary Data Fig. 1S). The carboxyl content of the oxidized cellulose was determined using conductometric titration, as described elsewhere (Besbes, Alila \& Boufi 2011). 


\subsection{Twin-screw extrusion (TSE) of pulp}

Samples were processed in a laboratory-scale co-rotating conical twin-screwDSMXplore15cc Micro-extruder, comprised of a clamshell barrel with a conical twin-screw extruder, which can be operated in batch and continuous modes ensured by a recirculation channel and a control valve built into the barrel. Pulps with a $10 \mathrm{wt} \%$ solid content were fed into the barrel and continuously extruded at a constant screw-speed of $200 \mathrm{rpm}$ via recirculation for $20 \mathrm{~min}$. The nanofibrillation yield was evaluated by centrifugation as follows: a dilute suspension with about $0.2 \mathrm{wt} \%$ solid content $(\mathrm{Sc})$ was centrifuged at 4500 rpm for $20 \mathrm{~min}$ to separate the nanofibrillated material (in the supernatant fraction) from the unfibrillated fibers which settled down. The fibrillation yield in $\%$ corresponds to the suspension concentration after centrifugation against the initial concentration.

\subsection{Processing TPS/CNF nanocomposites}

The processing route differed depending on whether ready CNFs or fibers were used:

$T P S / C N F-R$

When ready CNFs were used, starch granules (70 wt \%), glycerol (30 wt \%), CNFs (from 4 to $20 \mathrm{wt} \%$, on the basis of starch and glycerol together) and water (about half the weight of starch + glycerol) were pre-mixed manually, fed into the barrel and continuously extruded at $100 \mathrm{rpm}$, using the co-rotating conical twin-screw extruder, during $10 \mathrm{~min}$ at $25^{\circ} \mathrm{C}$ to ensure an effective dispersion of CNFs within the starch-glycerol mixture. Then, the temperature was progressively raised (during $10 \mathrm{~min}$ ) to $110^{\circ} \mathrm{C}$ while keeping the extrusion running at $200 \mathrm{rpm}$ to complete the gelatinization of starch. When the temperature reached $110{ }^{\circ} \mathrm{C}$, the extrusion was carried on for an additional $10 \mathrm{~min}$ to ensure an effective blending and water removal. Finally, a rectangular flat film was formed using a die with a rectangular crosssection of $10 \times 0.15 \mathrm{~mm}^{2}$. As an example, a TPS/CNF-R at $8 \mathrm{wt} \% \mathrm{CNF}-\mathrm{R}$ had the following composition: starch/glycerol/CNF: $64.4 / 27.6 / 80 \mathrm{~g}$. The CNFs are at $10 \mathrm{wt} \%$ solid content and the amount of added water was $45 \mathrm{~g}$.

\section{$T P S / C N F-F$}

When fibers were used, the extrusion process was as follows: starch granules (70 wt $\%$ ), glycerol $(30 \mathrm{wt} \%)$, fibers containing $50 \mathrm{wt} \%$ water were pre-mixed manually, fed into the barrel and continuously extruded at $100 \mathrm{rpm}$, using the co-rotating conical twin-screw extruder, during $15 \mathrm{~min}$ at a temperature of $25^{\circ} \mathrm{C}$. Then the temperature was progressively 
increased to $110^{\circ} \mathrm{C}$ (during $10 \mathrm{~min}$ ) and the extrusion was carried on at $200 \mathrm{rpm}$ during $15 \mathrm{~min}$ to complete the gelatinization of starch and the breakdown of fibers. A rectangular flat film was formed using a die with a rectangular cross-section of $10 \times 0.2 \mathrm{~mm}^{2}$. For the one-step extrusion, TEMPO-oxidized fibers were used with a content ranging from 2 to $20 \mathrm{wt} \%$. For comparison purpose, in one run, a composition with $10 \mathrm{wt} \%$ of neat fibers was tested to check for the occurrence of the disintegration of fibers during the extrusion process. As an example, a TPS/CNF-F at $8 \mathrm{wt} \%$ CNF-R has the following composition: starch/glycerol/oxidized fibers: 64.4/27.6/40 g. The oxidized fibers were at $50 \mathrm{wt} \%$ solid content and the amount of added water was $45 \mathrm{~g}$.

\subsection{Moisture absorption}

Extruded films were first dried by a hot-air oven at $90{ }^{\circ} \mathrm{C}$ for $12 \mathrm{~h}$ until reaching a constant weight and then placed in an environmental humidity chamber at $50 \%$ relative humidity $(\mathrm{RH})$ at a temperature of $23{ }^{\circ} \mathrm{C}$. The weight of the samples was recorded at regular intervals until a steady state value was reached. Three replicates were used for each composition.

\subsection{Tensile properties}

Tensile tests were carried out using an ARES rheometer (TA Instruments) equipped with an EVF measuring cell (TA Instruments) to maintain a constant-rate uniaxial extension in a strip specimen cut directly from the film produced by extrusion. A cross-head speed of $20 \mathrm{~mm} \cdot \mathrm{min}^{-1}$ was applied. All test specimens were conditioned at least for 3 days at $23{ }^{\circ} \mathrm{C}$ and $50 \% \mathrm{RH}$ to reach a stable water content. Five specimens were tested for each composition.

\subsection{Dynamic mechanical analysis (DMA)}

DMA experiments were conducted in tension mode using a PYRIS Diamond DMA (Perkin- Elmer, Waltham, MA, USA). Temperature scans were run from -80 to $120{ }^{\circ} \mathrm{C}$ at a heating rate of $2^{\circ} \mathrm{C} \cdot \mathrm{min}^{-1}$, a frequency of $1 \mathrm{~Hz}$ and an amplitude of $10 \mu \mathrm{m}$. Sample dimensions were about $20 \mathrm{~mm}$-long, $5 \mathrm{~mm}$-wide and $0.1-0.3 \mathrm{~mm}$-thick.

\subsection{Transparency measurement}

The transparency of the TPS-CNF films was measured at wavelengths from 200 to $800 \mathrm{~nm}$ using a Lambda 35UV-visible spectrometer from Perkin-Elmer. The transmission spectra of the films were recorded using air as reference. 


\subsection{Field-emission scanning electron microscopy (FE-SEM)}

Cross-sections of the extruded samples were prepared by fracturing the nanocomposite films in liquid nitrogen. The fracture surfaces were sputter-coated with a thin layer of $\mathrm{Pt}$ (about $1 \mathrm{~nm}$ ) and observed in a Zeiss Gemini 500 field-emission scanning electron microscope operating at $1 \mathrm{kV}$ with an in-lens secondary electron detector.

\subsection{Transmission electron microscopy (TEM)}

Droplets of dilute CNF suspensions were deposited onto glow-discharged carbon-coated copper grids and allowed to dry after negative staining with $2 \mathrm{wt} \%$ uranyl acetate. The specimens were observed with a JEOL JEM 2100-Plus microscope operating at $200 \mathrm{kV}$ and images were recorded using a Gatan Rio 16 camera.

\subsection{X-ray diffraction (XRD)}

Strips of films were X-rayed in reflection mode with a $\mathrm{CuK}_{\alpha}$ radiation $(\lambda=0.1542 \mathrm{~nm})$ generated in a Bruker AXS diffractometer (Madison, WI, USA) at $30 \mathrm{kV}$ and $100 \mathrm{~mA}$, from 5 to $70^{\circ}$, with a scanning step of $0.05^{\circ}$ and a step time of $10 \mathrm{~s}$.

\section{Results and discussion}

\subsection{CNFs produced by TSE}

CNFs used as reference were produced by TSE using TEMPO-mediated oxidized fibers from commercial eucalyptus pulps. The extrusion was run continuously at a solid content of $10 \mathrm{wt} \%$ during $20 \mathrm{~min}$ using a twin-screw mini compounder. The resulting CNF suspension had the aspect of a thick gel remaining stable for several weeks without any sign of phase separation or water syneresis. The fibrillation yield evaluated by centrifugation of dilute $\mathrm{CNF}$ suspension was found to be around $70 \mathrm{wt} \%$. This means that oxidized fibers were not fully disintegrated into CNFs and about $30 \mathrm{wt} \%$ of the suspension corresponded to micron-size particles encompassing partially fibrillated material as could be seen in (Supplementary Data Fig. S2). TEM images of the supernatant fraction clearly showed nanosized fibrils (Fig. 1), which agrees with our previous work (Baati, Magnin \& Boufi, 2017). 

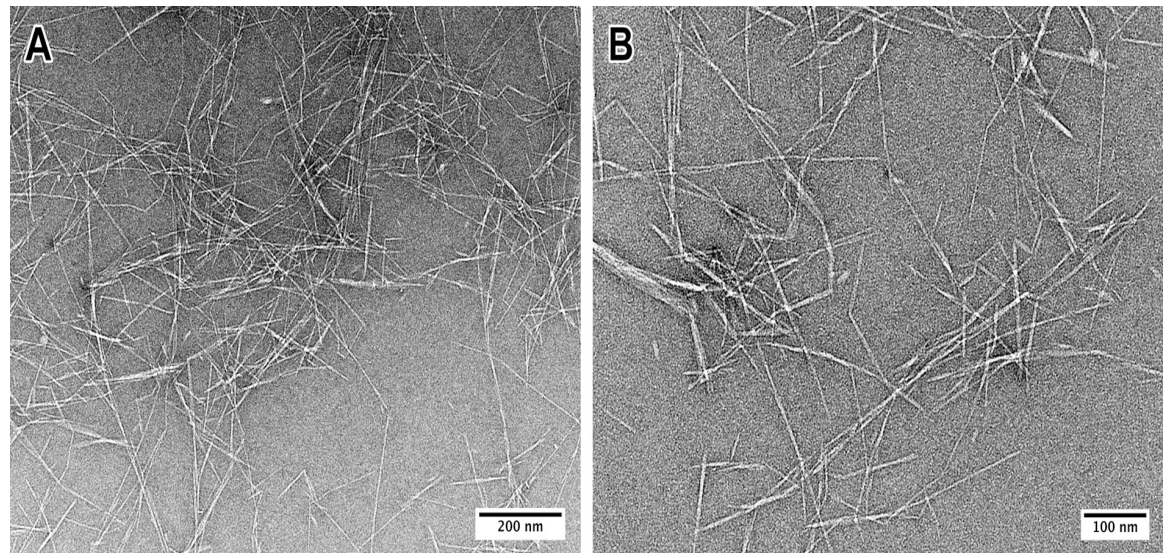

Figure 1. A,B) TEM images of negatively stained CNFs contained in the supernatant fraction produced by TSE of oxidized eucalyptus fibers.

\subsection{Mechanical properties of nanocomposites}

Typical stress-strain curves for the TPS/CNF films are shown in Fig. 2, and the results obtained from the tensile test using the mixing route with CNFs (labeled CNF-R) and in situ prepared TPS/CNF nanocomposite, starting from fibers (labeled CNF-F) are summarized in the form of histograms in Fig. 2B, 2C and 2D. Up to $6 \mathrm{wt} \% \mathrm{CNFs}$, no significant evolution of the strength and modulus was observed for both modes of preparation (ready CNFs and fiber extrusion), and over $8 \mathrm{wt} \%$, the modulus as well as strength increased steadily with increasing CNF content, with a stronger effect for nanocomposites produced in the presence of CNF-F. For instance, at a content of $15 \mathrm{wt} \% \mathrm{CNF}-\mathrm{F}$, the modulus/strength was 1.5/2 and 2.7/3.5 times higher than that of the unfilled TPS, respectively for CNF-R and CNF-F nanocomposites, respectively. The elongation at break progressively decreased with the addition of CNFs for both types of nanocomposites, from $22 \%$ for the neat TPS to about 10 and $14 \%$ nanocomposite with $15 \mathrm{wt} \% \mathrm{CNF}-\mathrm{R}$ and CNF-F, respectively.

The enhancement in strength and stiffness of the TPS nanocomposite produced via the in-situ route indicates the successful breakdown of the cellulose fibers into CNFs during the extrusion process. Moreover, the better mechanical properties for the in-situ processing mode in comparison to the addition mode of ready prepared CNFs might suggest a better breakdown of fibers into nanofibrils when the extrusion was performed in the presence of starch. However, if we compare the reinforcing effect brought by the addition of CNFs in TPS, with that observed with a soft acrylic or elastomer-like matrix, this enhancement in modulus and strength are modest. For example, in a nanocomposite based on an acrylic matrix and CNFs, prepared by casting, an increment in the storage modulus (E') ranging from 100 to 200 at $10 \mathrm{wt} \% \mathrm{CNF}$ content has been reported (Boufi \& Gandini, 2015). Two 
phenomenon are likely to account for this lower reinforcing effect in TPS matrix, as put forward by Anglès and Dufresne (2000), to explain the unexpected low reinforcing effect observed for tunicate/plasticized starch nanocomposites: (i) the plasticizing agents (glycerol and water) likely accumulate at the $\mathrm{CNF}$ /matrix interface which might reduce the possibility of interaction among neighboring fibrils through hydrogen bonding, and (ii) the similarity in the chemical structure of $\mathrm{CNF}$ and starch will favor a high interfacial interaction between the two phases at the expense of CNF-CNF interaction, which will reduce the strength of the nanocellulose network which is known to play a key role in the mechanism of nanocellulose reinforcement (Boufi, Kaddami \& Dufresne, 2014).
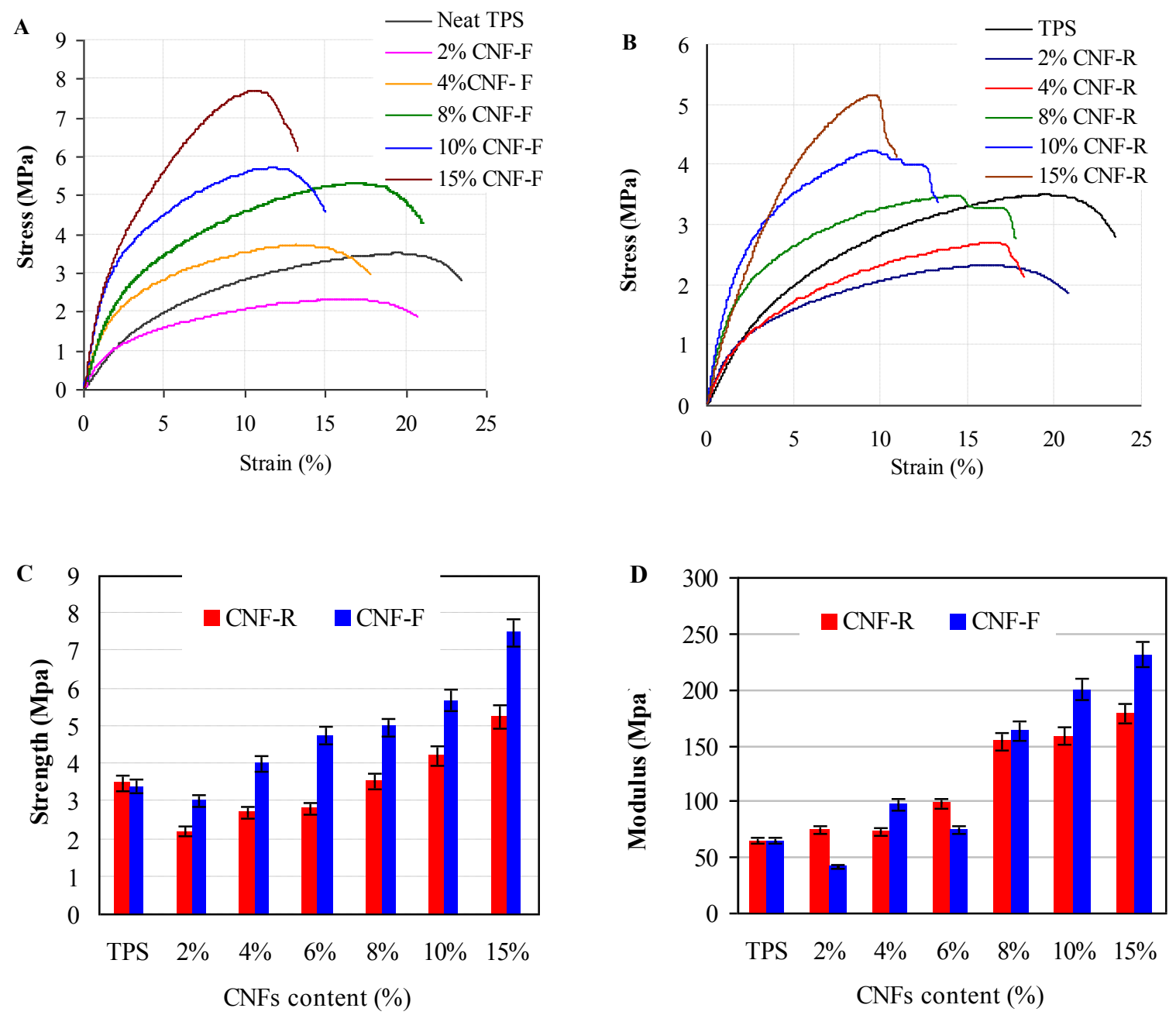

Figure 2. Mechanical properties of the TPS/CNF films at different CNF contents: (A, B) Typical stress-strain curves of TPS/CNF-R and TPS/CNF-F, (C) tensile strength and (D) Young's modulus. 

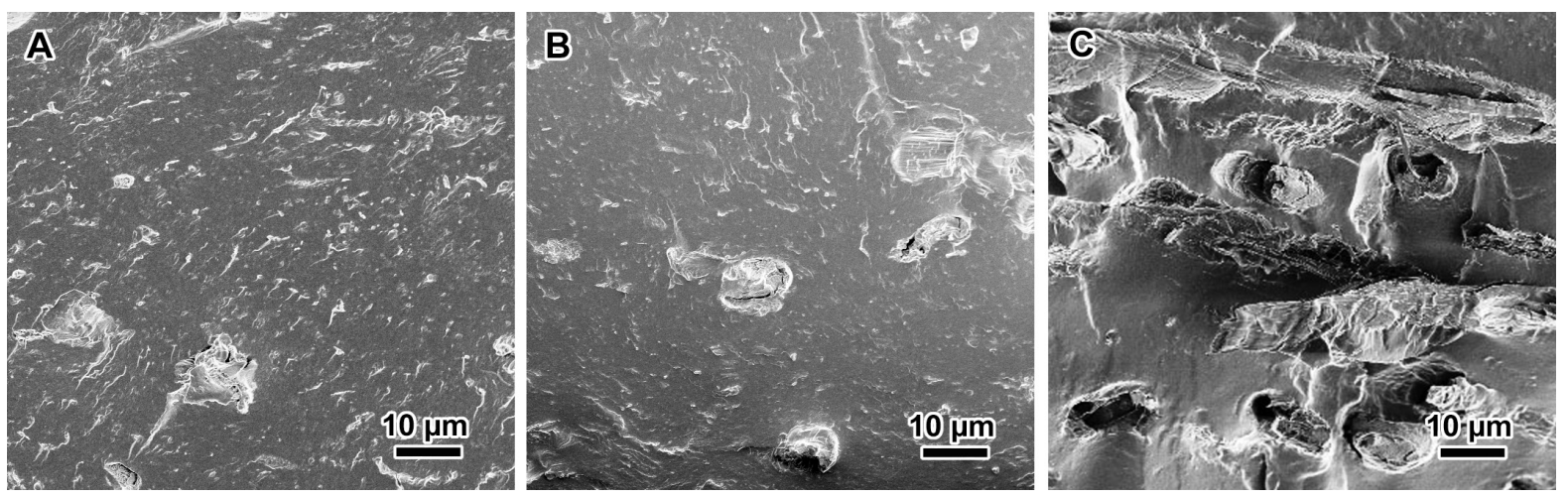

Figure 3. SEM images of surfaces of cryofractured composites: (A) TPS/CNF-R-10, (B) TPS/CNF-F-10, (C) TPS/fibers with10 wt $\%$ filler loading but without any fiber pretreatment.

\subsection{Morphology of TPS-CNF nanocomposites}

The SEM images from the cryofractured surfaces of the TPS/CNF nanocomposites containing $10 \mathrm{wt} \%$ CNFs produced via the mixing route and or the in-situ fibrillation (referred to as TPS/CNF-R-10 and TPS/CNF-F-10, respectively) are shown in Fig. 3. In TPS/CNF-R10 , the cross-section of the film is quite homogeneous with only rare micron-sized fibrils presumably corresponding to partially fibrillated material that has not been fully disintegrated during the production of CNFs (Fig. 3A). In TPS/CNF-F-10, the original fibers were mostly disintegrated with a fraction of micron-sized particles still present (Fig. 3B), presumably corresponding to fragments not fully broken down as seen for TPS/CNF-R. For the sake of comparison, the observation of a TPS composite film with containing $10 \mathrm{wt} \%$ untreated fibers produced following the same procedure was also included (Fig. 3C). The image clearly shows the presence of intact fibers with a diameter of around $10 \mu \mathrm{m}$ dispersed in the TPS matrix, some of them were pulled out and other were broken, without any sign of fibrillation. The huge difference in the heterogeneity of the sample and the size of dispersed particles are indicative of the successful breakdown of cellulose fibers during the one-step extrusion TSE with starch and glycerol.

\subsection{TEM analysis of the TPS-CNF suspension}

To get more evidence of the breakdown of the oxidized fibers during the extrusion process in the presence of TPS, TEM images were recorded from TPS/CNF-F-10 after dissolution of the nanocomposite film in hot water under reflux and dilution in water. More or less individualized CNFs can clearly be seen in the images of Fig. 4, intermingled with wormlike elements likely corresponding to retrograded amylose and/or amylopectin (Putaux, Buléon \& 
Chanzy, 2000). These networks sometimes seem to be attached to CNF bundles. However, it is not possible to say if this association is a remnant of the interaction between the constituents within the nanocomposite or if it results from the retrogradation after the solubilization and dilution of the material.
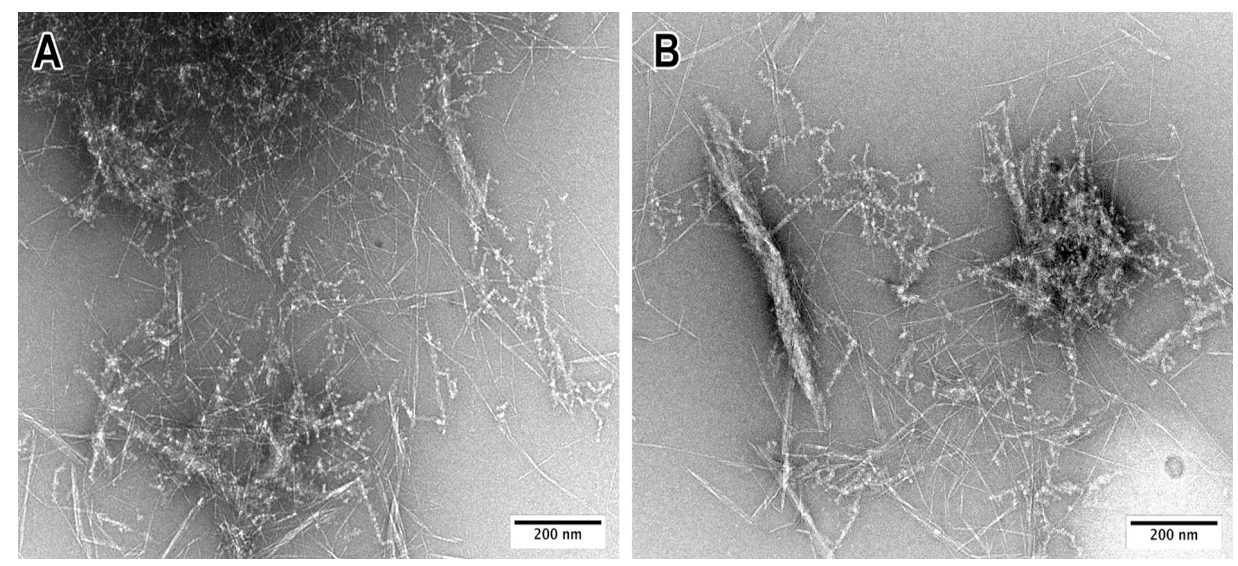

Figure 4. A,B) TEM images of a dilute suspension of TPS/CNF-F-10 after dissolution in water under reflux. The preparations have been negatively stained with uranyl acetate.

\subsection{Dynamic mechanical analysis (DMA)}

DMA analysis was also carried out to further investigate the evolution of the mechanical behavior of the materials over the temperature at different contents in CNFs. The evolution of the storage modulus (E') and loss modulus (E") of the TPS/CNF nanocomposites at different CNF contents are shown in Fig. 5. The neat TPS displayed a typical behavior of a partially miscible system with two main transitions. The first one around $-60^{\circ} \mathrm{C}$ (labelled $\beta$ ) is assigned to the glycerol-rich phase (Averous \& Boquillon, 2004) and is accompanied by an about 10-fold decrease in modulus. The second broad transition (labelled $\alpha$ ) extending from $10-50^{\circ} \mathrm{C}$ is associated with the glass transition of the starch-rich domains (Forssella et al., 1997) and the modulus is reduced by about 30 folds. In both TPS/CNF-R and TPS/CNF-F nanocomposites, the presence of CNFs resulted in an upward shift in the trace of E' which became more visible at 10-15 wt\% CNF content. The increment in $\mathrm{E}^{\prime}$ with respect to the neat matrix is much more marked over the glass transition of the starch-rich phase, which is a typical behavior of nanocellulose-based reinforcement. The comparison between TPS/CNF-F and TPS/CNF-R in this domain of temperature did not reveal any meaningful difference between the two processing routes in the magnitude of $\mathrm{E}^{\prime}$ in the temperature domain between 25 to $70{ }^{\circ} \mathrm{C}$, meaning that the stiffening effect brought by the inclusion of CNF is roughly the same whether the CNFs were added to TPS or produced in situ during the plasticization process. 

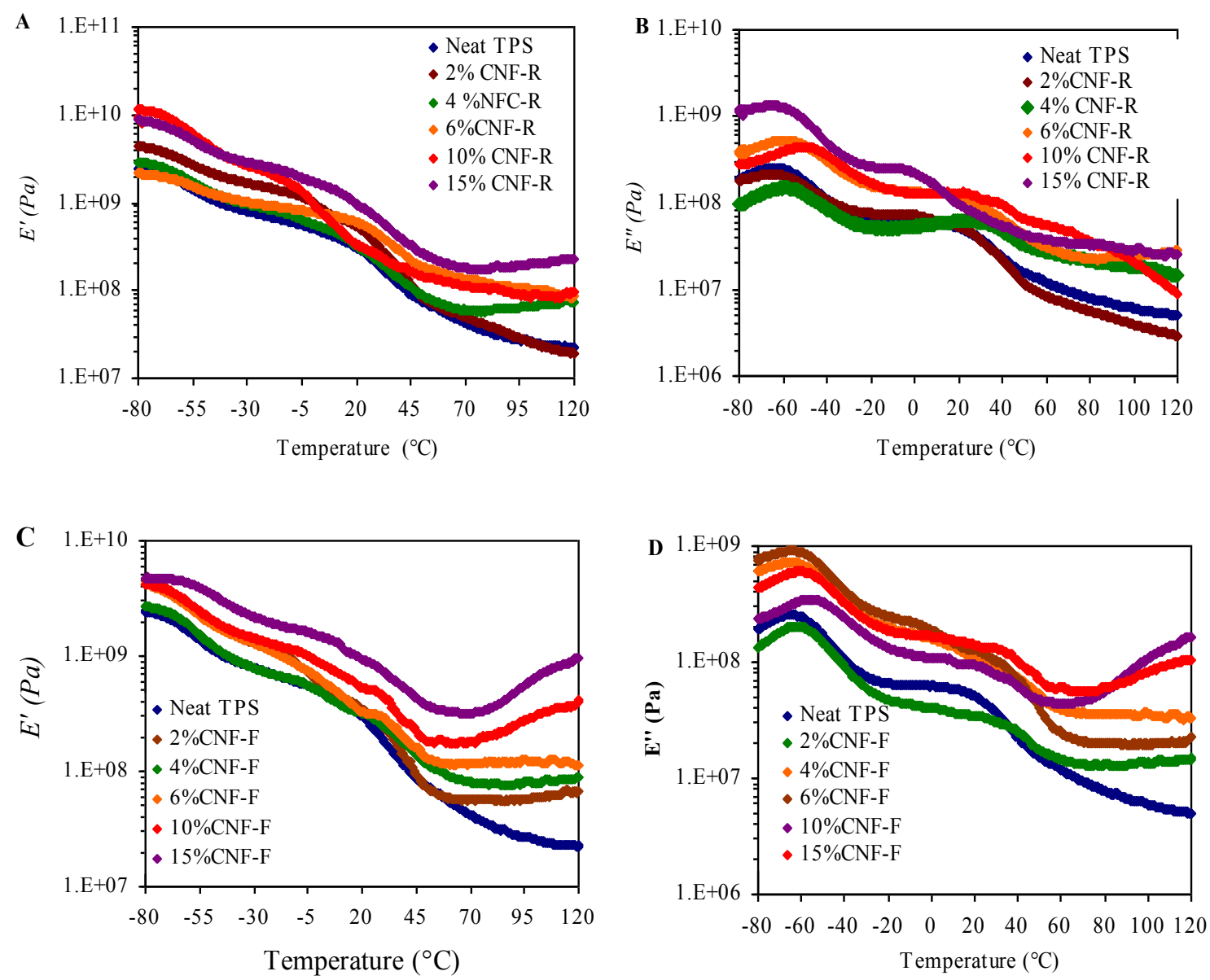

Figure 5. DMA analysis of TPS/CNF films: A,C) Storage tensile modulus, E' and (B) loss modulus E", versus temperature at $1 \mathrm{~Hz}$, using CNF-F and CNF-R fillers.

The position of the main relaxation $\alpha$ seems to be also affected by the incorporation of CNFs, shifting to higher temperature, especially when the CNF content exceeded $10 \mathrm{wt} \%$. This positive shift is indicative of reduced polymer chain mobility in the vicinity of CNFs confirming the high CNF-matrix interfacial interaction, presumably driven by hydrogen bonding between the surface hydroxyl groups of CNFs and starch/glycerol. This effective interfacial adhesion inevitably contributes to reduce the reinforcing potential of CNFs by hindering the set-up of hydrogen-bonded cellulose network within the matrix. This later is the main driving force for the strong reinforcing potential of nanocellulose-based fillers. This result supports the hypothesis made in section 3.1 regarding the origin of the lower reinforcing effect of CNFs in a TPS matrix. 


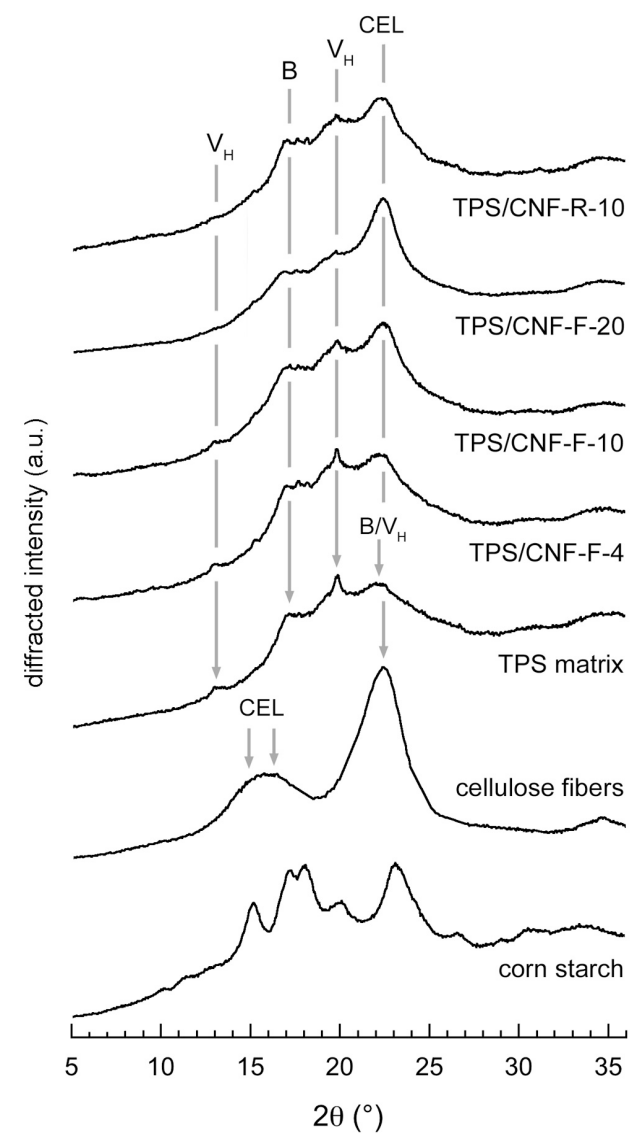

Figure 6. X-ray diffraction profiles of cellulose fibers, native corn starch, TPS, TPS/CNF-F and TPS/CNF-R at different filler contents, after storing for 15 days under $50 \% \mathrm{RH}$ at $23{ }^{\circ} \mathrm{C}$. The main peaks corresponding to cellulose (CEL), B-type starch and $\mathrm{V}_{\mathrm{H}}$ complexes are indicated.

\subsection{X-ray diffraction}

The XRD profiles of native corn starch, CNFs, TPS and of TPS/CNF nanocomposites at different CNF contents are shown in Fig. 6. The profile of neat fibers contains the diffraction peaks of native cellulose I with peaks at $2 \theta=14-16.5,22.5$ and $35^{\circ}$, while that of native corn starch granules is typical of A-type cereal starch with main peaks located at $2 \theta=15,17.3,18$ and $23.2^{\circ}$ (Lourdinet al., 2015). The profile of a neat TPS film does not contain any peak from A-type starch, proving that the initial starch granules have indeed been gelatinized during the thermoplasticization process. The profile contains rather broad peaks, which means that a large part of the starch matrix is amorphous or weakly crystalline, so their precise indexing is difficult. Two contributions may be recognized: (i) the peak at about $17-18^{\circ}$ would correspond to the B-type allomorph. However, the characteristic peak at $5.5^{\circ}$ is absent. Since B-type is known to be very sensitive to hydration, this absence may be both due to the low crystallinity of the matrix and an insufficient film hydration at 50\% RH. However, the 
presence of B-type was expected since this structure develops during the recrystallization (also called retrogradation) of gelatinized starch at room temperature (Lourdin et al., 2015); (ii) the peaks at 13-13.5 and $19.8^{\circ}$ would be ascribed to the $\mathrm{V}_{\mathrm{H}}$ allomorph that formed by recrystallization of amylose with endogenous lipids present in corn starch (Van Soest et al., 1996). The broad peak at about $22.2^{\circ}$ could be a superimposition of peaks from $B$ and $V_{H}$. There is no evidence of a crystalline complex formed between amylose and glycerol. As shown by Hulleman et al. (1996), if such complexes can indeed be prepared from a dilute solution of amylose, they form in drastically dehydrated conditions far different from those used during the processing of TPS/cellulose nanocomposites. In addition, the XRD peaks of the $\mathrm{V}_{\text {glycerol }}$ complex would differ from those of the $\mathrm{V}_{\mathrm{H}}$ allomorph.

The XRD profiles of TPS/CNF nanocomposites contain the typical diffraction peaks of the retrograded TPS matrix, $\mathrm{V}_{\mathrm{H}}$-type complexes and cellulosic filler. The contribution of the main cellulose peak at $22.5^{\circ}$ clearly increases with increasing CNF content. However, a reduction in the intensity of $\mathrm{V}_{\mathrm{H}}$ peaks seems to occur with the increase in the CNF content. A similar phenomenon was reported by Teixeira et al. (2011) in TPS plasticized with glycerol and reinforced with cotton nanofibers, which was explained by a possible decrease in the rearrangement of the starch chains due to the transcrystallization of amylose and amylopectin in the nanofiber surface.
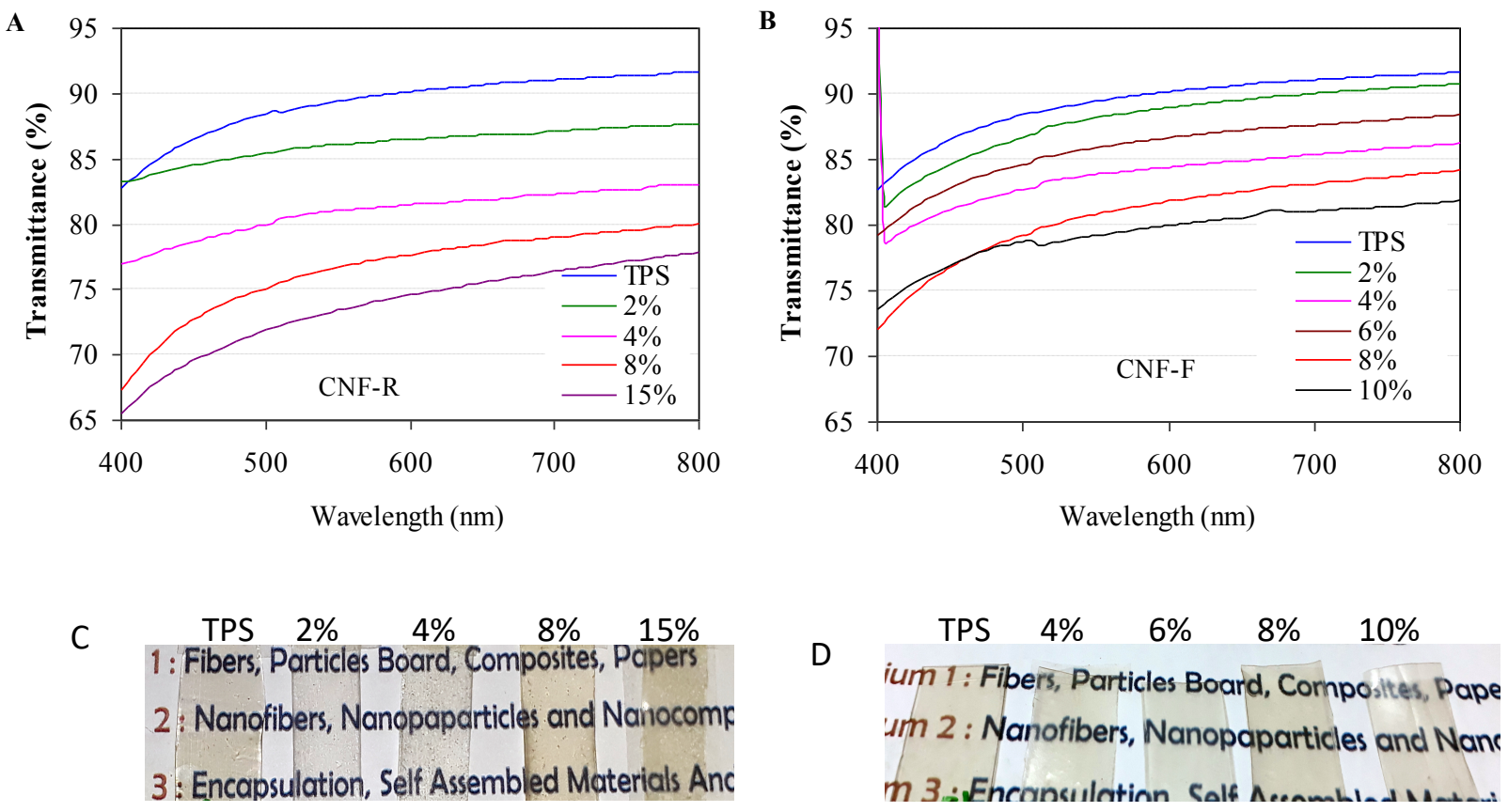

Figure 7. UV-Vis transmittance spectra for TPS/CNF films produced using CNF-R (A), and CNF-F(B)at different CNF contents. Visual aspect and transparency of TPS/CNF-R(C), and TPS/CNF-F at different content (indicated above the photo) (D). 


\subsection{Optical properties}

In general, the transparency of a nanocomposite is controlled by the size of the dispersed elements within the host matrix. Any aggregation of nanoparticles during processing and the presence of particle whose size exceeds $40 \mathrm{~nm}$ (corresponding to $\lambda / 10$ of the wavelength in the visible domain) results in a strong light scattering causing a reduction in transparency of the material. The optical transparency of TPS nanocomposite films was assessed by measuring the transmittance of the nanocomposite film in the visible domain (Fig. 7), with normalization to a $200 \mu \mathrm{m}$ thickness using the Beer-Lambert law. The unfilled TPS film was highly transparent with a $90 \%$ transmittance at $600 \mathrm{~nm}$. The addition of CNFs resulted in a steady drop in transparency. At $700 \mathrm{~nm}$, in comparison with the neat TPS, the transmittance was reduced by about 9,14 and $17 \%$ with addition of 4,8 and $15 \mathrm{wt} \%$ of CNF-R. The reduction was slightly lower for nanocomposites prepared by the in-situ route, reaching about 5,9 and $11 \%$ at similar content 4,8 and $15 \mathrm{wt} \%$ of CNF-F content, meaning that a better transparency in the nanocomposite film could be reached when CNFs were disintegrated in situ during the plasticization of TPS. This probably is due to the better dispersion of CNF within the TPS matrix when the in-situ disintegration route was adopted.

Further confirmation of the transmittance data could be obtained from the visual aspect against a background image of the TPS/CNF-R films with CNF content from 2 to $15 \%$ and TPS/CNF-F with fiber content from 4 to $10 \%$ (Fig. 7C and 7D). All films were transparent to translucent up to $15 \mathrm{wt} \% \mathrm{CNFs}$, without a significant reduction in the clarity of the films. In addition, no visible micron-sized cellulose fibrils could be seen by eye in any of the films.

A

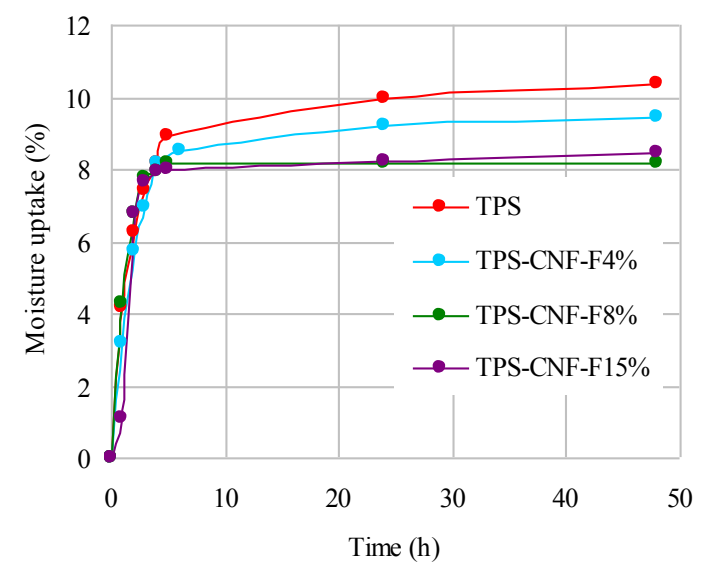

B

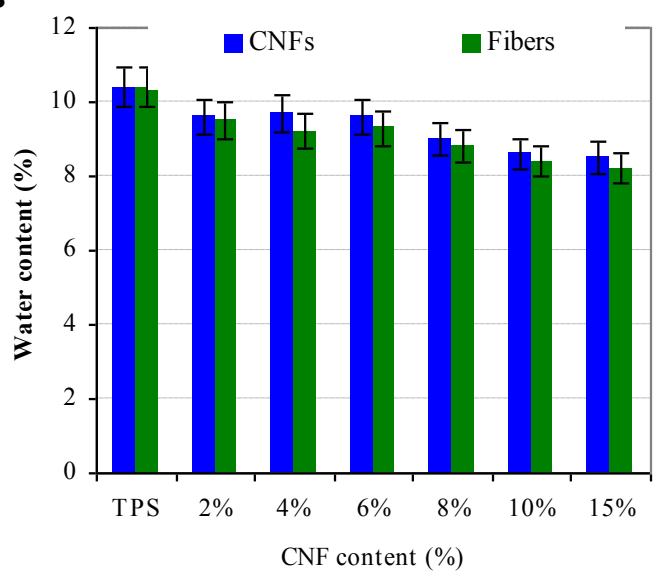

Figure 8. (A) Moisture uptake $v s$ time for TPS/CNF nanocomposite at different contents in CNFs, and (B) maximum water uptake according to CNF content. 


\subsection{Moisture absorption}

The moisture absorption maximum, at equilibrium and $50 \% \mathrm{RH}$, of $\mathrm{TPS} / \mathrm{CNF}$ nanocomposites with different CNFs content in is shown in Fig. 8. The moisture sorption maximum was slightly reduced by the addition of CNF for both CNF-R and CNF-F with the main reduction effect observed at $15 \mathrm{wt} \% \mathrm{CNF}$ content, decreasing from about $10 \%$ for unfilled TPS to about $8.5 \%$ for TPS/CNF-F. This result in agreement with those reported by Dufresne et al. concerning TPS/nanocellulose composites (Dufresne, Dupeyre \& Vignon, 2000) and is explained by the lower hydrophilic character of cellulose nanofibers compared to starch plasticized with glycerol starch due to higher degree of molecular order. No evolution of the water uptake was noted for both types of composite processing, which is expected since the water absorption in TPS-based polymer is determined essentially by the glycerol content.

The one-step production of nanocomposites based on fibrillated cellulose and a hydrophilic polymer has different advantages and shortcomings. As advantages, we can cite: (i) time saving since only a one-step process is used instead of two separate processes, (ii) avoid excessive dilution effect since high the amount of water added is less than if CNF at $1 \mathrm{wt} \%$ solid content is used, (iii) no need to use energy-consuming high-pressure homogenizer, grinder or the like process to produce firstly CNFs at low solid content $(<1 \mathrm{wt} \%)$. However, these approaches have some shortcomings:(i) necessity to use chemically pretreated fibers to make possible the breakdown of the cellulose fibers during the extrusion process; (ii) at this stage, the process is only valid for hydrophilic polymer matrices. More work is needed to extend this approach to other polymer matrices not soluble in water. We should emphasize that the present work is, to our knowledge, the first that reports the in-situ disintegration of pretreated cellulose fibers into CNFs during extrusion in the presence of a polymer matrix.

\section{Conclusion}

The aim of the present work was to produce TPS/CNF nanocomposites in a single step by twin-screw extrusion, using starch, glycerol and oxidized fibers as starting material. Unlike the conventional route for the processing of TPS/CNF nanocomposites where CNF suspensions were first prepared and then mixed with TPS or starch/plasticizer mixture, CNFs were presently generated in situ during the gelatinization of starch and the extrusion. This approach offers several advantages: (i) facilitate the processing of the nanocomposites, (ii) avoid the use of ready CNF suspension with low solid content, (iii) reduces the energy consumption during the generation of CNFs, (iii) reduction of the amount of water. TPS/CNF 
nanocomposites with a similar composition were produced by processing ready prepared CNFs, starch and glycerol for comparison purpose. The mechanical testing of nanocomposites showed an increase in tensile strength and modulus by the presence of CNFs becoming more marked as the CNF content exceeded $10 \mathrm{wt} \%$. Moreover, better mechanical performance was observed when the nanocomposite was CNFs were generated in situ during the extrusion process. The increase in modulus of the material due to the incorporation of CNFs was also confirmed by DMA, especially over the glass transition of the starch-rich phase. In addition, the TPS/CNF nanocomposites kept a good transparency quality of up to $20 \mathrm{wt} \%$ CNFs with only an $11 \%$ reduction in the transmittance in comparison of the unfilled TPS matrix, confirming the absence of micron-sized cellulose fibrils or aggregated CNFs. The effective disintegration of cellulose fibers during the extrusion of starch, glycerol and fibers was also confirmed by SEM and TEM

This new approach is the first report describing the single-route processing of TPS/CNF nanocomposites and the in-situ disintegration of cellulose fibers into nanofibrils. Work is in progress to extend this single step processing route of CNF-containing nanocomposites to other hydrophilic matrices such as polyvinyl alcohol.

\section{Acknowledgement}

This work has been partially supported by the LabEx Tec 21 (Investissements d'Avenir grant agreement \#ANR-11-LABX-0030). LRP and CERMAV are part of Institut Carnot PolyNat (Investissements d'Avenir - grant agreement \#ANR-11-CARN-030-01). The authors acknowledge the Glyco@Alps program (Investissements d'Avenir - grant agreement \#ANR15-IDEX-02) for financial support, and thank the NanoBio-ICMG Platform (FR 2607, Grenoble) for granting access to the Electron Microscopy facility. The FE-SEM images were recorded at the CMTC characterization platform of Grenoble INP supported by LabEx CEMAM (Investissements d'Avenir, grant agreement \#ANR-10-LABX-44-01). We thank Rachel Martin (CMTC) for the FE-SEM observations. The PHC-Utique program (19G1123) is also acknowledged. 


\section{References}

Alexandrescu, L., Syverud, K., Gatti A., \& Chinga-Carrasco G. (2013). Cytotoxicity tests of cellulose nanofibril-based structures. Cellulose 20,1765-1775.

Angles, M.N., \& Dufresne, A. (2000). A. Plasticized starch/tunicin whiskers nanocomposites. 1. Structural analysis. Macromolecules 33, 8344-8353.

Averous, L. (2004). Biodegradable multiphase systems based on plasticized starch: A review. Journal of Macromolecular Science, Part C Polymer Reviews 44(3), 231-274

Averous, L., \& Boquillon, N. (2004). Biocomposites based on plasticized starch: thermal and mechanical behaviours. Carbohydrate Polymers 56, 111-122.

Baati, R. Mabrouk, A.B., Magnin, A. \& Boufi, S. (2018). CNFs from twin screw extrusion and high pressure homogenization: A comparative study. Carbohydrate Polymers 195, $321-328$

Baati, R., Magnin, A., \& Boufi, S. (2017). High solid content production of nanofibrillar cellulose via continuous extrusion. ACS Sustainable Chemistry \& Engineering 5(3), $2350-2359$.

Barhoum, A., Li, H., Chen, M., Cheng, L., Yang, W., \& Dufresne A. (2018). Emerging applications of cellulose nanofibers, in "Handbook of Nanofibers", Springer, pp. 1-26.

Besbes, I., Alila, S., \& Boufi, S. (2011). Nanofibrillated cellulose from TEMPO-oxidized eucalyptus fibres: Effect of the carboxyl content. Carbohydrate Polymers 84, 975-983.

Boufi, S., \& Chaker, A. (2016). Easy production of cellulose nanofibrils from cornstalk by a conventional high-speed blender. Industrial Crops and Products 93, 39-47.

Boufi, S., \& Gandini, A. (2015). Triticale crop residue: a cheap material for high performance nanofibrillated cellulose. RSC Advances 5, 3141-3148.

Boufi, S., González, I., Delgado-Aguilar, M., Quim, T., \& Mutjé, P. (2016). Nanofibrillar cellulose as additive in papermaking process: A review. Carbohydrate Polymers 154, $151-166$.

Boufi, S., Kaddami, H., \& Dufresne, A. (2014). Mechanical performance and transparency. Macromolecular Materials and Engineering 299, 517-638.

Dufresne, A. (2017). Nanocellulose: From Nature to High Performance Tailored Materials. Walter de Gruyter GmbH \& Co KG.

Dufresne, A., Dupeyre, D. \& Vignon, M.R. (2000). Cellulose microfibrils from potato tuber cells: Processing and characterization of starch-cellulose microfibril composites. Journal of Applied Polymer Science 76, 2080-2092. 
Dufresne, A. \& Vignon, M.R. (1998). Improvement of starch film performances using cellulose microfibrils. Macromolecules 31, 2693-2696.

Forssella, P.M., Mikkilti, J.M., Moates, G.K.,\& Parker, R. (1997). Phase and glass transition behaviour, of concentrated barley starch-glycerol-water mixtures, a model for thermoplastic starch. Carbohydrate Polymers 34, 275-282.

Hietala, M., Mathew, A.P., \& Oksman K. (2013). Bionanocomposites of thermoplastic starch and cellulose nanofibers manufactured using twin-screw extrusion. European Polymer Journal 49, 950-956.

Ho, T.T.T., Abe, K., Zimmermann, T., \& Yano, H. (2015). Nanofibrillation of pulp fibers by twin-screw extrusion. Cellulose 22, 421-433.

Hulleman, S.H.D., Helbert, W., \& Chanzy, H. (1996). Single crystals of amylose complexed with glycerol. International Journal of Biological Macromolecules 18, 115-122.

Kaushik, A., Singh, M., \& Verma, G. (2010). Green nanocomposites based on thermoplastic starch and steam exploded cellulose nanofibrils from wheat straw. Carbohydrate Polymers 82, 337-345

Lee, K.Y, Aitomäki, Y., Berglund, L.A., Oksman, K., \& Bismarck, A. (2014). On the use of nanocellulose as reinforcement in polymer matrix composites. Composites Science and Technology 105, 15-27.

Lourdin, D., Putaux, J.-L., Potocki-Véronèse, G., Chevigny, C., Roland-Sabaté, A., \& Buléon, A. (2015). Crystalline structure in starch. In "Starch - Metabolism and Structure" Nakamura Y. ed., Springer Japan, pp. 61-90.

Martins, I.M.G., Magina, S.P., Oliveira, L., Freire, C.S.R., Silvestre, A.J.D., Neto, C.P., \& Gandini A. (2009). New biocomposites based on thermoplastic starch and bacterial cellulose. Composites Science and Technology 69, 2163-2168.

Nechyporchuk, O., Belgacem, M.N., \& Bras, J. (2016). Production of cellulose nanofibrils: A review of recent advances. Industrial Crops and Products 93, 2-25.

Nechyporchuk, O., Pignon, F., \& Belgacem, M.N. (2015). Morphological properties of nanofibrillated cellulose produced using wet grinding as an ultimate fibrillation process. Journal of Materials Science 50(2), 531-541.

Putaux, J.-L., Buléon A., \&Chanzy, H. (2000). Network formation in dilute amylose and amylopectin studied by TEM. Macromolecules 33, 6416-6422.

Rol, F., Karakashov, B., Nechyporchuk, O., Terrien, M., Meyer, V., Dufresne, A., et al. (2017). Pilot scale twin screw extrusion and chemical pretreatment as an energy efficient 
method for the production of nanofibrillated cellulose at high solid content. $A C S$ Sustainable Chemistry \& Engineering 5, 6524-6531.

Tarrés, Q., Saguer, E., Pèlach, M., Alcalà, M., Delgado-Aguilar, M., \& Mutjé, P. (2016). The feasibility of incorporating cellulose micro/nanofibers in papermaking processes: the relevance of enzymatic hydrolysis. Cellulose 23,1433-1445.

Teixeira, E. de M., Lotti, C., Corrêa, A.C., Teodoro, K.B.R., Marconcini, J.M., \& Mattoso, L.H.C. (2011). Thermoplastic corn starch reinforced with cotton cellulose nanofibers. Journal of Applied Polymer Science 120, 2428-2433.

Teixeira, E. de M., Pasquini, D., Curvelo, A.A.S., Corradini, E., Belgacem, M.N., \& Dufresne, A. (2009). Cassava bagasse cellulose nanofibrils reinforced thermoplastic cassava starch. Carbohydrate Polymers 78, 422-431.

Van Soest, J.J.G., Hulleman, S.H.D., de Wit, D., \& Vliegenthart, J.F.G. (1996). Crystallinity in starch bioplastics. Industrial Crops and Products 5, 11-22.

Vikman, M., Vartiainen, J., Tsitko, I., \& Korhonen P. (2015). Biodegradability and compostability of nanofibrillar cellulose- based products. Journal of Polymers and the Environment 23, 206-215. 


\section{Supplementary data}

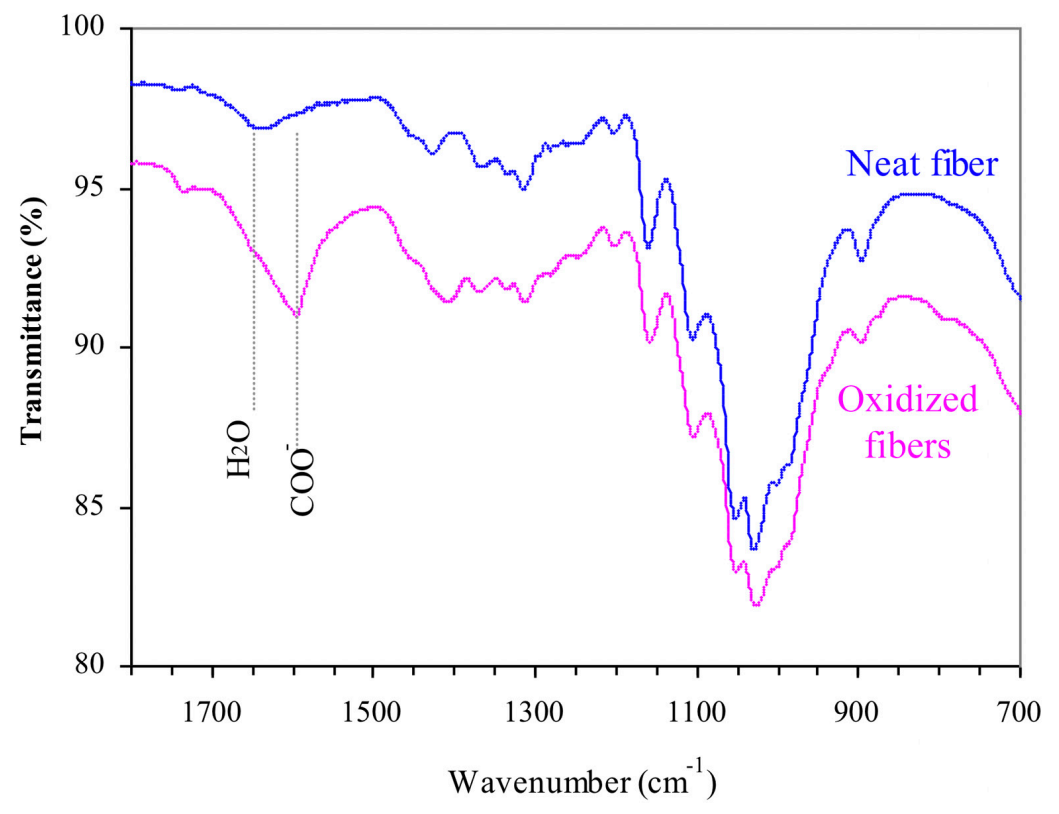

Figure 1S : FTIR spectra of neat and oxidized fibers. The oxidized fibers were characterized by a large band around $1600 \mathrm{~cm}^{-1}$ typical of carboxylate groups.
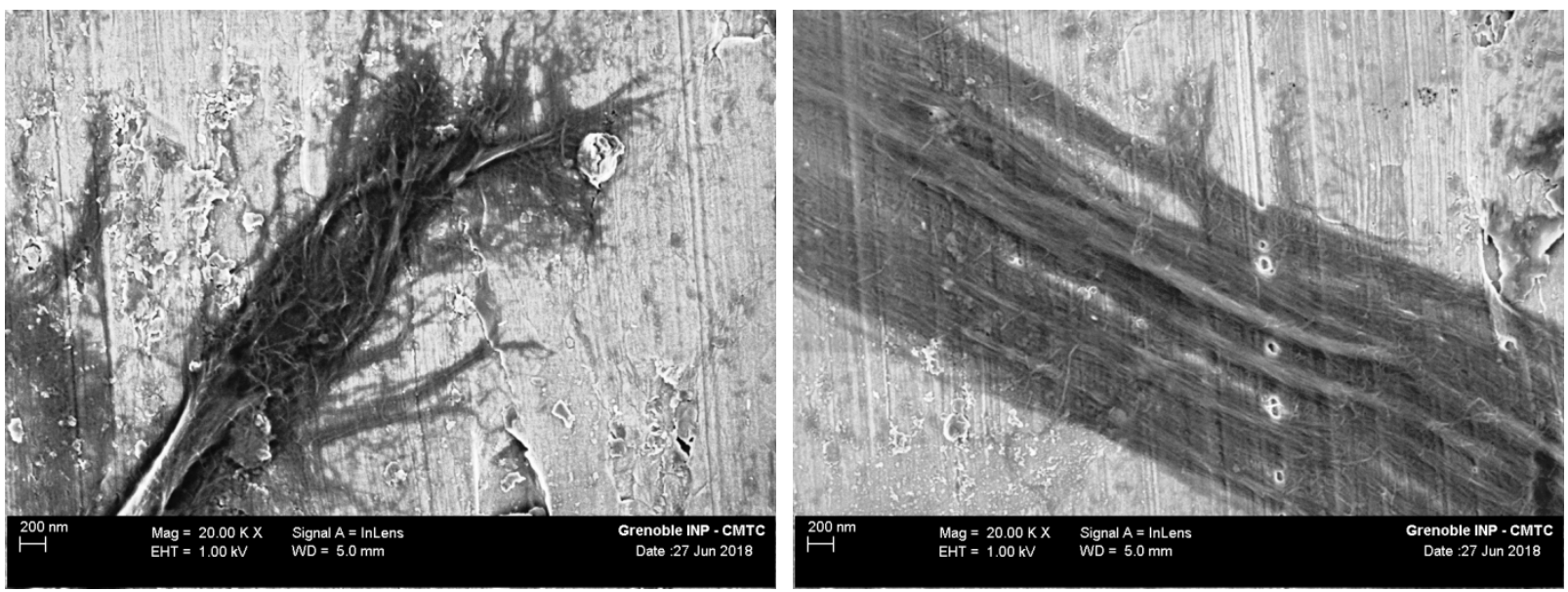

Fig. 2S: FE-SEM observation of sediment fraction corresponding to partially fibrillated material. 